\title{
Comparison of quota sampling and stratified random sampling
}

\begin{abstract}
The possibility that researchers should be able to obtain data from all cases is questionable. There is a need; therefore, this article provides a probability and non-probability sampling.

In this paper we studied the differences and similarities of the two with approach that is more of fritter away time, cost sufficient with energy required throughout the sample observed. The pair shows the differences and similarities between them, different articles were reviewed to compare the two.

Quota sampling and Stratified sampling are close to each other. Both require the division into groups of the target population. The main goal of both methods is to select a representative sample and facilitate sub-group research. There are major variations, however. Stratified sampling uses simple random sampling when the categories are generated; sampling of the quota uses sampling of availability. For stratified sampling, a sampling frame is necessary, but not needed for quota sampling. More specifically, stratified sampling is a method of probability sampling which enables the calculation of the sampling error. For quota samples, this is not possible.
\end{abstract}

Quota sampling is therefore primarily used by market analysts rather than stratified sampling, as it is mostly cost-effective and easy to conduct and has the appealing equity of satisfying population reach. However, it disguises potentially significant bias.

Keywords: stratified sampling, quota sampling, sampling of non-probability, probability sampling
Volume I0 Issue I - 202 I

\author{
Rufai lliyasu, Ilker Etikan \\ Department of Biostatistics, Near East University Faculty of \\ Medicine, Nicosia-TRNC, Cyprus
}

Correspondence: Ilker Etikan, Department of Biostatistics, Near East University Faculty of Medicine, Nicosia-TRNC, Cyprus, Email ilker.etikan@neu.edu.tr

Received: January 13,2021 | Published: February 25, 202 I

\section{Introduction}

Research is a systematic and objective attempt to study the problem for finding the solution. The sampling and assignment of the sampling method is essential in cases where the researcher is unable to count because of the time constraints and cost. Sampling has two main form, Probability Sampling and non-probability sampling, and the full population list is required in probability sampling while non-probability sampling the list of population is not required for the sampling process. All the mentioned sampling techniques are used for different purpose. The probability sampling technique is a successful sample selection technique, and there is a fair chance of sample selection for all members of the population. This is a strong representative of the population while their cost is more and it consumes more time for the study and the report is submitted in the long time to concerned department while the non-probability sampling techniques are not the good representative of the population but its cost and time is less and in time the research is processed and report is submitted. In stratified sampling the strata are prepared and according to proportion sample is selected, similarly in quota sampling data are divided into different quotas and sample is taken.

\section{Sampling of probability}

In probability sampling, a total sample has an equal chance of being chosen. In large part, we can conclude that the probability sample is the probability that each segment of the population has a probability of being selected that is not null. This sampling method gives the likelihood of our sample being representative of the population.

It is mostly used in quantitative research if you need to generate results that are representative of the entire population, you need to apply probability sampling procedure. We have many probability sampling methods but in this case we are going to compare stratified random sampling.

\section{Sampling with non-probability}

Non-probability sampling uses non-randomized methods, to pull the sample, unlike sampling with probability. Non-probability sampling technique generally takes in judgment. Participants are chosen since they are easy to access in its place of randomization. Your friends and course mate, for instance, have a superior opportunity to be among your sample. While non- probability sampling is a simple and useful method of selecting a sample in some cases, the method is correct and in some cases, the only method available. One of the key drawbacks of the non- Probability sampling is results that have been shown to lack generality via this method. Although the results obtained by this method normally refer to the group studied, it may be incorrect to expand these findings beyond that specific sample. We may learn some basic phenomena throughout the non-probability method, with the potential to generate useful insights. We researched surviving theoretical observations or generating new ones in the non-probability sample. This method of sampling is considered less difficult, less cost, and simple to apply as matched to probability sampling. Also in this case of non-probability sampling we are to compare quota sampling. ${ }^{1-3}$

\section{Stratified Sampling}

This is a sampling which involve chosen some group of items from population based on classification and random selection. It involves 
separating the target population element in to homogenous, mutually exclusive segment, from each segment simple random sampling is chosen. The selected sample from different strata is combined to have a single sample. Stratified sampling is the technique of probability sampling in which the characteristics of a precise variable are interpreted in the universe relative to this variable. For Example, if we considered a sample of 100 people in a universe of 500 people based on sex variable, and the population consist of 200 females and 300 males there should be 60 males and 40 females. ${ }^{4}$

In stratified random sampling there are two main types of proportionate stratified sampling and disproportionate stratified random sampling.

\section{Proportionate sampling of stratified}

This is one of the two stratified sampling types. The number of elements assigned to the different strata is proportional to the representation of the strata in the target population. In proportionate terms. That is to say, in the target population, the scope of the sample taken from each stratum is proportional to the relative size of that stratum. Each stratum is smeared with exactly the same sampling fraction, giving each single element inside the population in the target population. Each stratum is smeared with exactly the same sampling fraction, giving any single element within the population an equal chance of being picked. Then a self-weighting sample is the resulting sample.

\section{Stratified sampling disproportionate}

This is another stratified sampling technique. The number of elements sampled in disproportional stratified random sampling from each stratum is not equal to their population representation. In the population, the element has no fair chance of being included in the sample. There is no smearing of the fraction with each stratum. On the other hand, there are distinct sampling fractions in the strata. To estimate population variables, the population arrangement must be applied as weights to compensate for the disproportionality in the sample. On the other hand, for some research activities, disproportionate stratified sampling may be more appropriate than proportionate stratified sampling. ${ }^{5-9}$

\section{Quota sampling}

This is one of the most common sampling methods for nonprobability. Sampling is performed before a specific number of units is chosen for many sub-populations. Therefore, as there are no guidelines as to precisely how these quotas should be completed, for some sub-populations, quota sampling is definitely a way of achieving sample size purposes. In probability sampling methods, quota sampling is similar to stratified sampling. However, unlike stratified sampling, quota sampling has no capacity to represent the universe.

Unquestionably, amongst the type of probability sampling quota sampling methods has advantages over the rest, it is a less expensive and appropriate method to easily generate a sample because it is stressfree to administer and there is no need for a sample frame. Quota sample advocates are not tired of disputing that a stratified random sampling arrangement using elaborate and very stringent quotas can be calculated; so many fine-grained so-called quota controls such as age, gender, education, social status, occupation, city and region size can be used.
Quota sampling is a process that starts with the universe description, then creates certain quotas about the unit structures that will be in the sampling, and selects individuals to fit in with these quotas. ${ }^{10}$

Although quota sampling has its drawbacks, the accuracy and objectivity of administrative data source depends on the reliability and validity of quota controls. In addition, the probability of biased collection of respondents within each cell would not be eradicated even by quota controls. In such a scenario, this is dependent on the nature of the situation in the interview. as interviewer, do not press hard on a single case and instead go for the most cooperative respondents. Moreover, it is often difficult to check whether a respondent is 'creatively' redefined to meet a giving quota by interviewers rather than having to make even more cells.

\section{Proportional sampling of quota}

If you want to represent the key characteristics of the population, sample a proportional quantity of each of them. For Example, if you know that the population is 60 percent male and 40 percent female, and you need of 100 percent total sample size, you will sample continuously until you reach those numbers and then you will stop. So, if you already have 40 women for your survey, but not the 60 men, you will continue to sample men, but even if real women respondents come along, you will continue to sample men, because you acquired it already "met the quota." The issue here is that you have to decide on the particular features on which the quota will be the quota will be based. Is it going to be by age, gender, religion, education etc.

\section{Non-proportional quota sampling}

In this process, you can indicate the minimum number of sampled units you want in each group. You're not concerned about having figures that fit the proportions of the population at this juncture. As an option, you just want to provide enough to ensure that even small group in the community can be spoken about. This approach is the non-probabilistic counterpart of stratified in that it is usually used to ensure that there is adequate representation of smaller groups in the sample.

\section{The features of quota sampling:}

These are the ten features of quota sampling are given below.

I. It aims to achieve the best representation in the final sample of respondents.

II. Quotas replicate the desires of the population in a real sense. More representative are the estimates made.

III. Quota samples differ in consistency

IV. It saves time for the collection of study data as the sample reflects the population.

V. It saves research costs if the quotas represent the population correctly.

VI. The number of types of people taking the survey is tracked.

VII. The investigator often splits the population into subgroups.

VIII. The full population is reflected by the survey.

IX. The sampling approach is used by researchers to classify the characteristics of a particular group of individuals. 


\section{More representative are the estimates made.}

\section{An example of quota sampling:}

Let's look at a fundamental instance of quota sampling:

A researcher wants to survey people about what brand of smartphones they want to use. He/she finds 500 respondents to be a sample size. He/she is also only involved in surveying ten US states. This is how a researcher can segment the population by quotas:

$\triangleright$ Gender: 250 boys and 250 girls

$\triangleright$ Age: 100 respondents aged 16-20, 21-30, 31-40, 41-50, and 51+ each.

$\triangleright$ Job status: 300 people working and 200 people unemployed.

(Further nested quotas are applied by researchers. For example, out of the 200 unemployed individuals, 150 must be students.)

$\triangleright$ Location: 50 per-state responses

The researcher may submit quotas based on the sampling frame, depending on the type of study. The researcher is not expected to divide the quotas equally. As per his/her requirement, he/she divides the quotas (as shown in the example where the researcher interviews 300 employed and only 200 unemployed individuals). To reach out to the respondents, random sampling may be performed.

\section{Difference between stratified sampling and quota sampling}

Quota sampling, organized together in identical units, is somewhat similar to that of stratified sampling. However, the way the units are selected varies, Units are picked at random in stratified random sampling, but quota sampling is usually left to the interviewer to determine who to sample. This process in a selection bias.

The main goal of both methods is to select a representative sample and facilitate sub-group research. There are major variations, however. Stratified sampling uses simple random sampling when the categories are generated; sampling of the quota uses sampling of availability. For stratified sampling, a sampling frame is necessary, but not needed for quota sampling.

The table below shows an example of each technique, nothing of the advantages and limitations of each, we will describe the population as approximately 1000 students in al'niima academy as all middle school children. ${ }^{11-16}$

\begin{tabular}{llll}
\hline $\begin{array}{l}\text { Sampling } \\
\text { techniques }\end{array}$ & Example & Advantages & Limitations \\
\hline $\begin{array}{l}\text { Stratified random } \\
\text { sampling }\end{array}$ & $\begin{array}{l}\text { All I, } 000 \text { names are placed in the computer database and arranged according to grades } \\
\left(6^{\text {th }}, 7^{\text {th }}, 8^{\text {th }}\right) \text {. The computer is then told to select } 35 \text { names from each of the three grades } \\
\text { at random. Such kids and their parents have been contacted. }\end{array}$ & $\begin{array}{l}\text { Representative } \\
\text { of the } \\
\text { population }\end{array}$ & $\begin{array}{l}\text { May be difficult to } \\
\text { obtain the list may } \\
\text { be more expensive }\end{array}$
\end{tabular}

The investigator prefers the Ist 20 6th grade boys, the Ist 6th grade girls, the Ist 20 7th grade boys, the first 7th grade girls, the Ist 8th grade boys, and the Ist 8th grade

Quota Sampling

$\begin{array}{ll}\text { Simple, Easy, } & \text { Population } \\ \text { convenient } & \text { might not be } \\ \text { No complete } & \text { representative. }\end{array}$

\section{Comparison of quota sampling and stratified sampling}

\begin{tabular}{|c|c|}
\hline Stratified Sampling & Quota Sampling \\
\hline \multicolumn{2}{|l|}{ Quota sampling and Stratified sampling similarities } \\
\hline $\begin{array}{l}\text { The population is divided into categories; element from } \\
\text { each category are then chosen. }\end{array}$ & $\begin{array}{l}\text { The population is divided into categories; element from each } \\
\text { category are then chosen. }\end{array}$ \\
\hline $\begin{array}{l}\text { The aim is to select a representative sample and promote } \\
\text { analyses by subgroup. }\end{array}$ & $\begin{array}{l}\text { The goal is to pick a representative sample and encourage subgroup } \\
\text { analyses. }\end{array}$ \\
\hline \multicolumn{2}{|c|}{ Quota sampling and Stratified sampling Dissimilarities } \\
\hline This is a probability sampling method. & This is a non-probability sampling method. \\
\hline A sampling frame is needed. & Does not need sampling frame. \\
\hline Bias Selection is being minimized. & Bias Selection cannot be minimized. \\
\hline Has the proficiency to represent the universe & Has no proficiency to represent the universe \\
\hline It is possible to estimate random sampling errors. & Unable to predict random sampling errors. \\
\hline
\end{tabular}




\section{Conclusion}

The stratified random sampling is the good technique for the selection of sample, and there is a chance for each member of the population to pick sample. It is a good representative of the population while their cost is more and it consumes more time for the study and the report is submitted in the long time to concerned department while the Quota sampling are not the good representative of the population but its cost and time is less and in time the research is processed and report is submitted. In stratified sampling the strata are prepared and according to proportion sample are selected, similarly in quota sampling data are divided into different quotas and sample is taken.

\section{References}

1. Etikan I, Bala K. Sampling and sampling methods. Biom Biostat Int J. 2017;5(6):215-217

2. Daniel J. Selecting the form of probability sampling. SAGE Journal Publications. ISBN: 9781452272047.

3. Etikan I, Musa SA, Alkassim RS. Convenience sampling and purposive sampling comparison. American theoretical and applied statistics Journal. 2016;5(1):1-4.

4. Bora semiz Buket. Stratified sampling and Quota sampling comparison in terms of population mean. The $7^{\text {th }}$ MAC 2016 Proceeding. 2016.

5. Etikan I, Alkasim R, Abubakar S. Snowball Sampling and Sequential sampling technique comparison. Int J Biom Biosta. 2016;3(1):00055.

6. Khan Naushad, Mahnoor Naushad, Ayasha Akbar, et al. Critical Review of COVID-2019 in Pakistan and Its Impact on Pakistan Economy. Agriculture University, Peshawar-institute of Developmental studies. 2020;1-32.
7. Statistics Canada, Sampling methods date modified 2017-10-23.

8. Nayeem showkat, Huma Parveen. Aligarh Muslim University. Nonprobability and probability sampling chapter. 2017.

9. Wisniowski A, Joseph W Sakshaug, Diego Andres Perez Ruiz, et al. Integrating survey inference probability and Non-probability samples. Survey Statistics journal. 2020;8(1):120-147.

10. Spencer P, Kubaschewski O. A thermodynamic assessment of the ironphosphorus system. Archiv für das Eisenhüttenwesen. 1978; 49(5): 225 228.

11. Yang Keming, Banamah Ahmad. Sampling of quotas as an alternative to sampling of probabilities? An Experimental studies Online sociological research. 2014;19(1):29.

12. Hamed Taherdoost. Sampling method in research methodology. International Journal of Academic Research in Management. 2016;5(2):1-11.

13. Prof. John Adwok, Nairobi Hospital, P.O Box 21274-00505, Nairobi, Kenya.

14. Ilker Etikan, Sulaiman Abubakar Musa, Rukayya Sunusi Alkassim. Comparison of Convenience Sampling and Purposive Sampling. American Journal of Theoretical and Applied Statistics. 2016;5(1)1-4.

15. Geo H Brown. A comparison of sampling methods. Journal marketing. 1947;11(4):331-337.

16. Stephen F, McCaerthy P. Sampling opinions. John Wiley and sons, New York, USA; 1958. 